\title{
Proteomics approach as a new way to predict tenderness as compared to the classical South African Beef Carcass Classification System
}

\author{
K.W. Moloto ${ }^{1 \#}$, L. Frylinck ${ }^{1}$, P.E. Strydom ${ }^{1} \&$ G. Koorsen ${ }^{2}$ \\ ${ }^{1}$ Animal Production Institute, Agricultural Research Council, Private Bag X2, Irene 0062, South Africa \\ ${ }^{2}$ University of Johannesburg, Faculty of Science, Department of Biochemistry, PO Box 524, \\ Auckland Park, 2006, South Africa
}

(Received 31 July 2014; Accepted 3 March 2015; First published online 29 July 2015)

\author{
Copyright resides with the authors in terms of the Creative Commons Attribution 2.5 South African Licence. \\ See: http://creativecommons.org/licenses/by/2.5/za \\ Condition of use: The user may copy, distribute, transmit and adapt the work, but must recognise the authors and the South African \\ Journal of Animal Science.
}

\begin{abstract}
The current South African Beef Carcass Classification System classifies carcasses using physical attributes such as age, fatness code and the conformation of the carcass as the only indicator of tenderness, implying that optimal tender meat is obtained from carcasses from animals with no permanent teeth. Research shows that if we take modern technologies (use of beta-agonists, growth hormones and electrical stimulation amongst others) into account, this is not necessarily true. In this study $\mathrm{m}$. longissimus lumborum samples were collected from Nguni breed animals, snap frozen and stored at $-80{ }^{\circ} \mathrm{C}$ for further use in search of protein markers that can be used to predict tenderness in a non-invasive manner. Proteins were extracted in $1 \mathrm{~mL}$ TES buffer, isoelectrically focused on strips ( $\mathrm{pH} 5-7$ ) and separated by 2D SDS PAGE, stained with coomassie brilliant blue G250) and imaged by a Chemi-doc Mp imager. The results showed differences in protein expression, e.g. some proteins are present at 1 hour post mortem (Day 0) but absent at 3 days post mortem (Day 3). Other proteins showed an increase in expression as ageing progresses. These proteins correlated with myofibril fragments and Warner Bratzler changes which were measured. There are several factors that may be responsible for these changes in differential expression during ageing such as proteolytic action (i.e. calpains and calpastatin system, cathepsins) or maybe apoptosis. The proteins in the profiles of Nguni breed animals which showed differential expression in response to ageing are still to be determined.
\end{abstract}

Keywords: Nguni breed, protein markers for tenderness, two dimensional gel electrophoresis (2D SDS PAGE)

\# Corresponding author: Molotok@arc.agric.za

\section{Introduction}

Tenderness is regarded as the main quality attribute of meat. Therefore it puts quality assurance systems under pressure to guarantee consistently tender meat. The current South African Carcass Classification System (SABCCS) classifies carcasses based on age, fatness code and the conformation of the carcass. It uses age as the only indicator of tenderness implying that optimal tender meat is obtained from carcasses from animals with no permanent teeth (A-class). Research shows that if we take modern technologies (use of beta-agonists, growth hormones and electrical stimulation amongst others) into account, this is not necessarily true (Strydom, 2011). Seeing that age does not guarantee tenderness, the classification system sometimes disadvantages farmers who produce animals with 2 to 4 permanent teeth, which produce meat that may be similar or even more tender than the meat of A-class animals produced from modern feedlots. Therefore the current SABCCS is limited and new ways and means should be investigated to improve the reliability of the SABCCS to identify tender meat.

According to a recent survey by the Animal Production Institute of the Agricultural Research Council (ARC-API), tenderness is one of the most variable and inconsistent quality characteristics as measured from a representable sample of meat products in the retail sector (Strydom, 2013). Tender meat is obtained when all role players in the meat industry manage various factors including genetics, nutrition, growth promoters, 
pre-harvest stress, harvest technology (electrical stimulation, chilling), post-harvest conditions (duration of shelf life or ageing, packaging, temperature) and cooking (Schönfeldt \& Strydom, 2011).

There is currently no single non-invasive method to predict meat tenderness of a carcass specifically at abattoir level in order to grade or classify the carcass or cut according to eating quality.

Two-dimensional gel electrophoresis (2-DE) is a useful technique for identifying differentially expressed proteins that are associated with meat quality. Such differentially expressed proteins could be used as molecular biomarkers for meat quality and may provide new insights into the molecular mechanisms and pathways related to marbling and tenderness (Hollung et al., 2007). Proteomics is probably one of the best tools to achieve this. Currently, this is a very complicated technique but through perseverance, it could be simplified and equipment could be developed so that such a technique will be used in a common classification system.

At the Animal Production Institute (ARC-API) of the Agricultural Research Council meat scientists are searching for protein markers that are related to tenderness and other characteristics that can be used as predictors of the above mentioned attributes. Such differentially expressed proteins could be applied as molecular biomarkers for meat quality and may provide understanding into the molecular mechanisms and pathways related to e.g. marbling and tenderness. Protein markers, individually or a group of proteins, will be identified by statistically comparing their expression with attribute related measurements determined in the $m$. longissimus lumborum, such as Warner Bratzler shear force (WBS), sarcomere length, myofibril fragmentation (MFL) and calpain system (tenderness related), water holding capacity and drip loss (juiciness related), etc. Identified protein markers will be tested on unrelated samples to verify their efficacy as markers for meat quality attributes.

This paper describes protein expression related to tenderness detected in 2D SDS PAGE analyses of the indigenous South African Nguni breed collected from $m$. Iongissimus lumborum.

\section{Materials and Methods}

Twenty indigenous South African Sanga Nguni animals fed a normal feedlot diet were slaughtered at the ARC-IRENE abattoir. Samples were collected on different days post-slaughter (Day 0 within 1 hour postslaughter, Day 3 and Day 14 post-slaughter) snap frozen with liquid nitrogen and stored at $-80{ }^{\circ} \mathrm{C}$ until further analysis. The frozen muscle $(200 \mathrm{mg})$ was homogenised in $1 \mathrm{~mL}$ TES buffer and extracted according to method of Jia et al. (2006). Protein concentrations were measured with a commercial kit at $750 \mathrm{~nm}$ (RC-DC Protein Assay, Bio-Rad) in an ELX Universal micro-plate reader with BSA as a standard. Individually extracted samples were analysed by means of 2D SDS PAGE. Each sample was run in triplicate to evaluate the technical variability.

Protein separation in the first dimension was performed on an immobilised $\mathrm{pH}$ gradient (IPG) strips (Bio-Rad), $24 \mathrm{~cm}$, spanning the pH region $5-7$. Proteins $(700 \mu \mathrm{g})$ were loaded onto each IPG strip by in-gel rehydration overnight at room temperature. Isoelectric focusing (IEF) was performed using the Ettan IPGPhor II unit (GE Healthcare BioSciences, Uppsala, Sweden) and using a stepwise programme described as follows: $500 \mathrm{~V}$ for $2 \mathrm{~h}$, increase to $1000 \mathrm{~V}$ for $2 \mathrm{~h}$, increase to $10000 \mathrm{~V}$ for $3 \mathrm{~h}, 10000 \mathrm{~V}$ for $7: 36 \mathrm{~h}$ (seven hour thirty six minutes). The second dimension proteins were separated on $12 \%$ SDS-PAGE using the Ettan DALT six large format vertical system (GE Healthcare Bio-Sciences). Gels were stained with Coomassie brilliant blue $\mathrm{G} 250$.

Gels were imaged using Chemi-doc Mp (Bio-Rad Hercules, CA, USA) equipped with Image Lab software. Comparative analysis of the expressed proteins of two samples within the breed was carried out using PDQuest Advanced 2D analysis software. Each sample had three gel replicates. Gels were normalised using the group consensus tool. Differentially expressed protein spots were statistically significant using the student's t-test at the 95\% significant level. (www.bio-rad.com/en-us/product/pdquest-2d-analysis-software)

Tenderness was measured mechanically by means of Warner Bratzler shear force.The myofibril fragments from filtrate derived extracts from LL were examined with an Olympus BX40 system microscope using a 400X magnification. 100 Myofibril fragments of each sample were measured, using a software package (analySIS Life science) (Snyman et al., 2008).

\section{Results}

Two-dimensional electrophoretic analyses were performed in biological triplicates on protein extracts from $m$. longissimus lumborum samples. Triplicates increased the significance of the statistics supporting spots showing differential photo densities. Figure $1 \mathrm{~A}$ shows intact proteins expression in an Nguni sample taken within 1 hour post slaughter (Day 0) and Figure 1B also shows proteins expression in an Nguni sample 


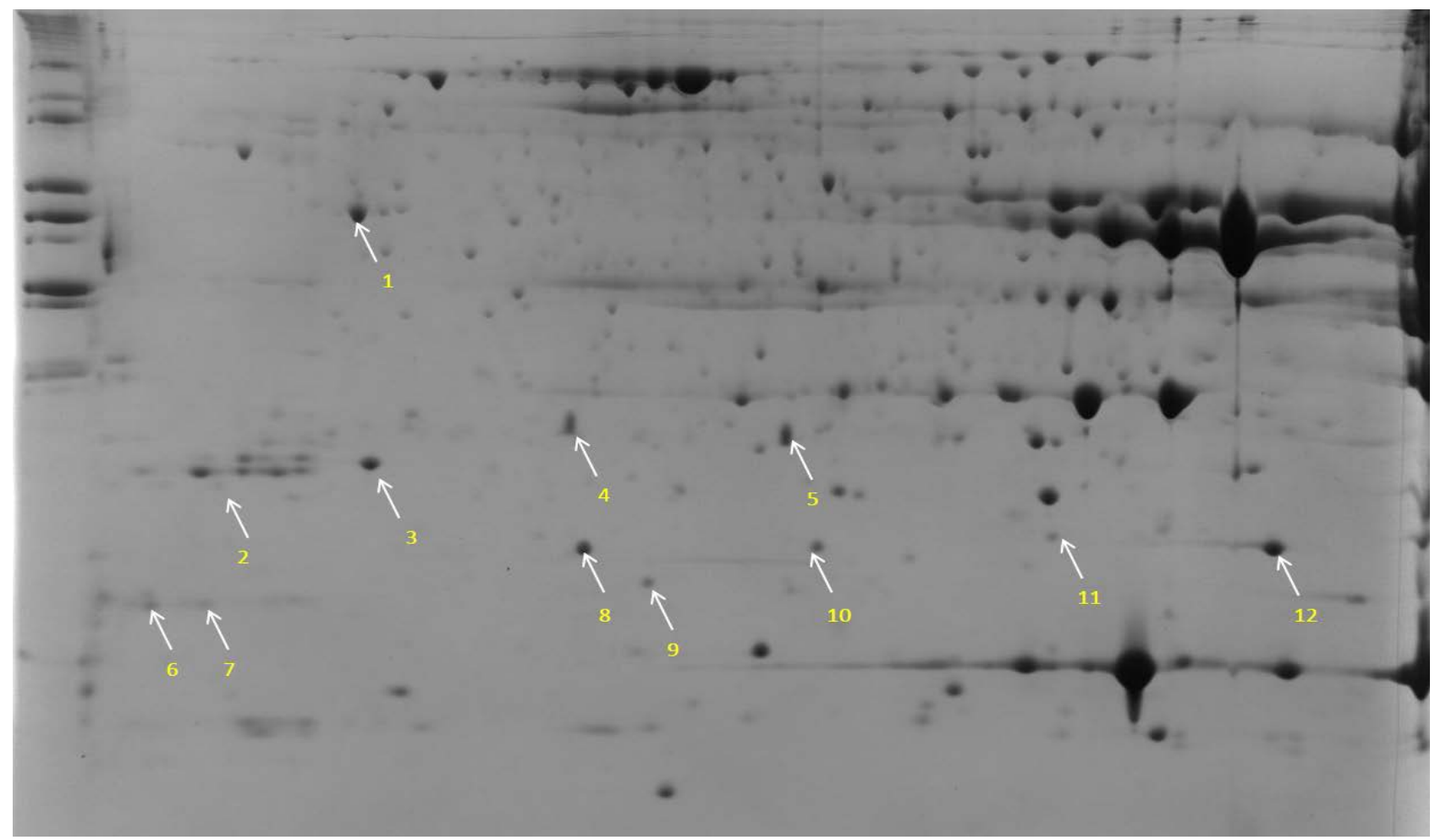

Figure 1A Gels from a Nguni sample showing differences in protein expression on Day 0 (zero).

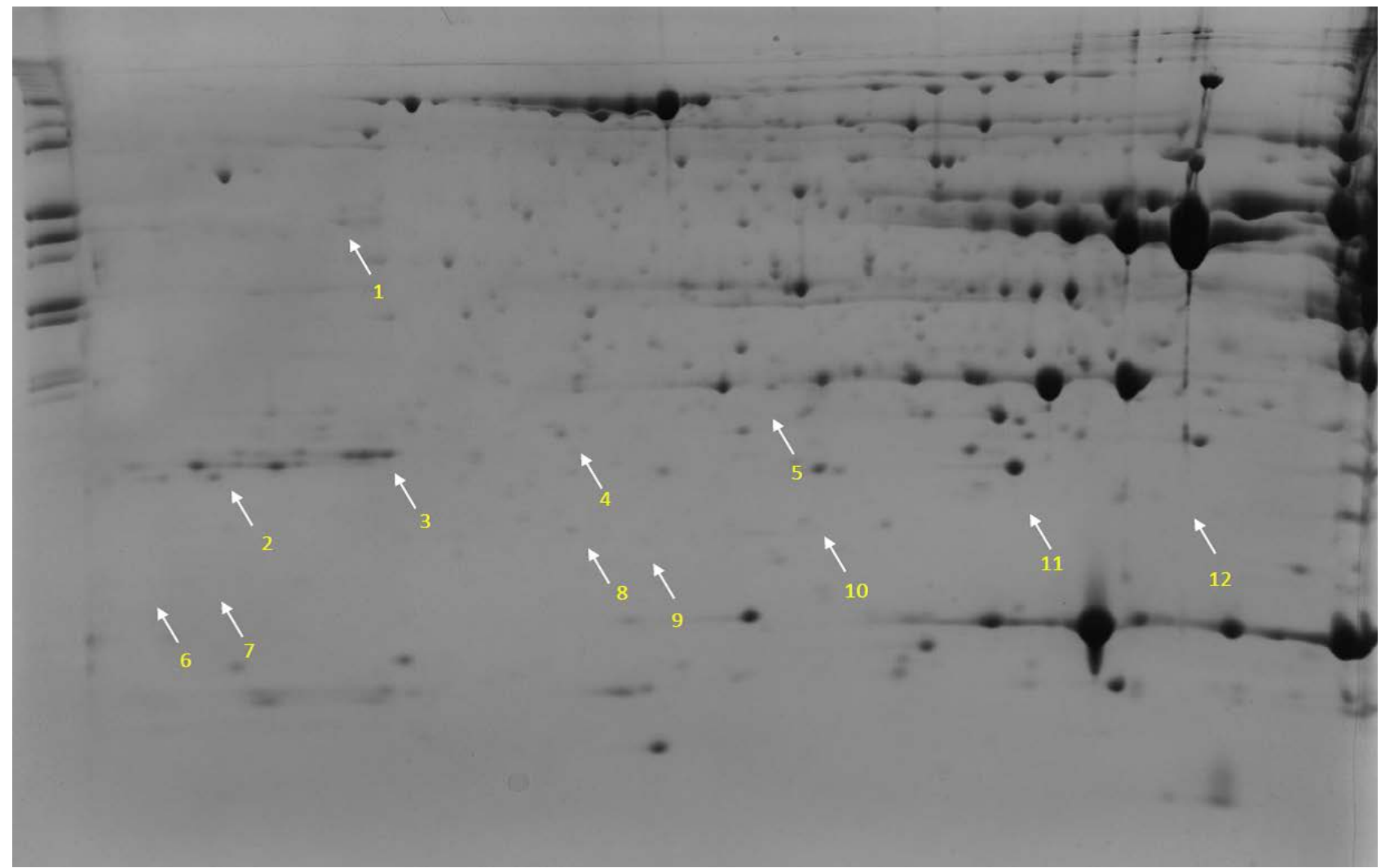

Figure 1B Gels from a Nguni sample showing differences in protein expression on Day 3.

Figure 1 Gels from a Nguni sample showing differences in protein expression on day zero and day three. Gel A shows proteins present on day zero and gel B shows proteins which are absent on day zero and that are present on day three. 
taken three days after slaughter (Day 3). There are several proteins present on Day 0 but absent on Day 3; these proteins are marked with white arrows. The general Figure1A shows more protein spots than Figure $1 \mathrm{~B}$.

The protein spots indicated in Figure 2 were statistically significant using student's t-test at $95 \%$ significance level. Figure 2A shows a protein spot which is absent on Day 0 but as ageing progress the spot starts to appear as seen on Day 3 and Day 14. For this study the molecular range of the proteins was not determined but the $\mathrm{pH}$ range was identified between $5-7$. The graphic and computer based image analysis of the 2DE gels help to pinpoint the differentially expressed proteins as ageing progresses. The quantity/expression of the Spot a on Day 3 and Day 14 are almost similar suggesting that ageing from Day 3 to Day 14 did not have much effect on Spot a. Spot $b$ in Figure 2B shows a high expression level on Day 0 but little on Day 3 and Day 14. The expression of Spot $b$ is similar to Spot $a$ in the same Figure 2A with the
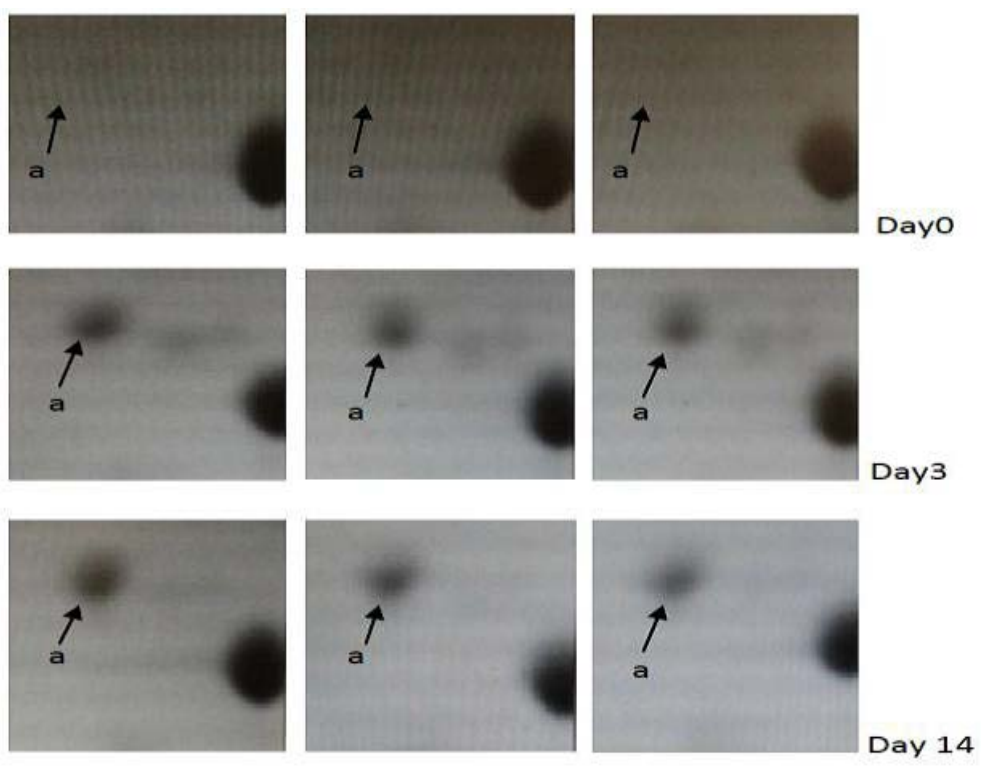

\section{Figure 2A}
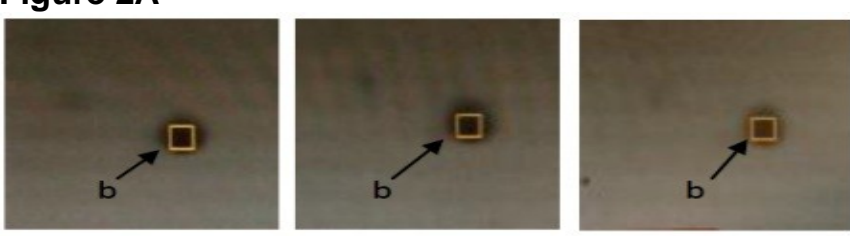

Day 0
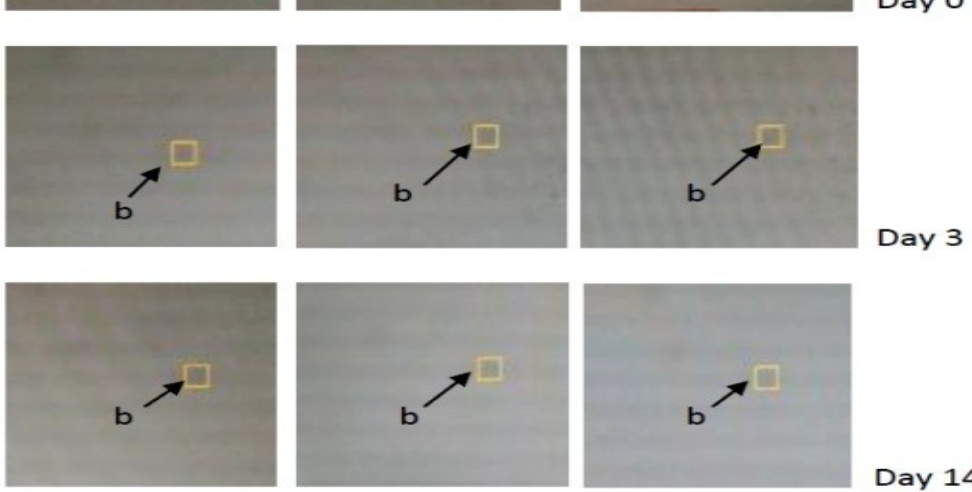

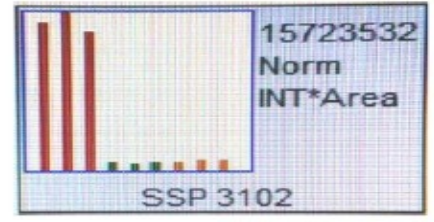

Figure 2C

\section{Figure 2B}

Figure 2 A and B. An enlarged section of representative spots showing differential expression patterns after several days of ageing at $0-4^{\circ} \mathrm{C}$. IEF pH range is $5-7,12 \% \mathrm{~T} 3 \% \mathrm{C}$ Acrylamide. Gels have been stained with Coomassie brilliant blue G250. Figure 2C. The expression of the spots in Figure B is also shown in PDQuest software bar graphs to illustrate the effect of ageing. 
difference that Spot a is not present on Day 0. The expression of Spot $b$ is also shown on the PDQuest generated bar graph in Figure 2C. As can be seen on the bar graph the expression levels of Spot b are highly expressed on Day 0. The spots expression seems to relate WBS and MFL to tenderness shown in Table 1. The WBS decreases with ageing with a value of $6.37 \mathrm{~kg}$ at Day 3 and $4.67 \mathrm{~kg}$ at Day 14 . The same pattern is found for the MFL which is $35.37 \mu \mathrm{m}$ at Day 3 and $22.53 \mu \mathrm{m}$ at Day 14. The names and properties of these proteins still need to be determined by mass spectrometry.

Table 1 Average Warner Bratzler shear force (WBS) and myofibril fragment length (MFL) measured in animal N50 $\mathrm{m}$. longissimus lumborum showing decreases as ageing progresses

\begin{tabular}{lcc}
\hline Characteristics & N50 & N50 \\
& Day 3 & Day 14 \\
\hline WBS $(\mathrm{kg})$ & 6.37 & 4.67 \\
MFL $(\mu \mathrm{m})$ & 35.73 & 22.53 \\
& & \\
\hline
\end{tabular}

\section{Discussion}

In this introductory investigation the protein patterns and their post mortem behaviour (Figures $1 \mathrm{~A}$ and $1 \mathrm{~B}$ ) in relation to ageing were studied. The expression patterns of 2DE gels reveal proteins present in Figure $1 \mathrm{~A}$, but absent in Figure 1B. There may be several reasons which can explain this absence. As ageing progresses proteins may undergo autolysis and yield different products. Another scenario might be that the action of enzymes degrade the proteins and form different products. Additional mechanisms, such as apoptosis, have also been proposed to influence the proteome and the meat quality. Also proteins involved in the energy metabolism tend to degrade as ageing progress (Di Luca et al., 2013). With the use of PDQuest, differential expression was made possible. In this study several proteins (43 spots) have shown differential expression in relation to ageing. For example in Figure 2A Spot a is not detected on Day 0, but fully detected during the ageing progress. Conversely Figure $2 \mathrm{~B}$ shows that $\mathrm{Spot} b$ is highly expressed on Day 0 , but expression decreases as ageing progresses. There are several factors that may be responsible for these changes in differential expression, such as ageing because of proteolytic action (calpains and calpastatin system and or cathepsins) (Lonergan, et al., 2010). Intermediate $\mathrm{pH}$ affects the calpains and cathepsins negatively as they are less optimal in that $\mathrm{pH}$ range. After slaughter there are many changes taking place in the carcass when the muscle changes to meat. As muscle goes into rigor, there is a loss of extensibility and along with that, a change in the texture of the meat (Polati et al., 2012). There are more than a few key proteins that are modified during post mortem ageing such as actin and myosin. Those proteins are located in different regions of the muscle cell, and most have been implicated in some way as being vital in maintaining the structure and function of the muscle cell. Several studies focused on investigating these proteins as biomarkers for tenderness. Searching for biomarkers related to tenderness has presently gained much interest in the field of meat science. A biomarker is a characteristic that is objectively measured and assessed as a pointer of normal biologic responses to interference (Tambor et al., 2010). It has to be able to fulfil certain criterion such as unbiased diagnosis.

\section{Conclusion}

In this study several proteins have shown a change in response to ageing such as proteins marked in Figure $1 \mathrm{~A}$ and $1 \mathrm{~B}$ and proteins $\mathrm{a}$ and $\mathrm{b}$ in Figure 2. Current results shows that as the meat becomes more tender (decrease in shear force in Table 1) the protein pattern changes. In the next phase of the study, these proteins will be identified with mass spectrometry and their role in meat tenderness will be evaluated.

\section{Acknowledgements}

The authors wish to express their gratitude to the following committees of institutions: The Agricultural Research Council (ARC) for facilities and financial support. The Red Meat Research and Development of South Africa and the Technology and Human Resources for Industry Programme of the Department of Trade and Industry, South Africa for the funding of this project.

\section{References}

Di Luca, A., Elia, G., Mullen, A.M., Hamill, R.M., 2013. Monitoring post mortem changes in porcine muscle through 2-D DIGE proteome analysis of Longissimus muscle exudate. Proteome Science 11, 9. 
Hollung, K., Veiseth, E., Jia, X., Færgestad, E.M. \& Hildrum, K.I., 2007. Application of proteomics to understand the molecular mechanisms behind meat quality. Meat Sci. 77, 97-104.

Jia, X., Hollung, K., Therkildsen, M., Hildrum, K.I. \& Bendixen, E., 2006. Proteome analysis of early postmortem changes in two bovine muscle types: $\mathrm{m}$. longissimus dorsi and $\mathrm{m}$. semitendinosis. Proteomics. 6, 936-944.

Lonergan, E.H., Zhang, W. \& Lonergan, S.M., 2010. Biochemistry of post mortem muscle — Lessons on mechanisms of meat tenderization, Meat Sci. 86, 184-195.

Ohlendieck, K., 2011. Skeletal muscle proteomics: current approaches, technical challenges and emerging techniques. Skeletal Muscle 1, 6.

Polati, R., Menini, M., Robotti, E., Millioni, R., Marengo, E., Novelli, E., Balzan, S. \& Cecconi, D., 2012. Proteomic changes involved in tenderization of bovine Longissimus dorsi muscle during prolonged ageing. Food Chem. 135, 2052-2069.

Schönfeldt, H.C. \& Strydom, P.E., 2011. Effect of age and cut on cooking loss, juiciness and flavour of South African beef. Meat Sci. 87, 180-190.

Snyman, J.D., Frylinck, L. \& Strydom, P.E., 2008. Comparison of two methods to determine myofibril fragmentation post mortem. 54th International Congress of Meat Science and Technology, 2008,10 15 August, Cape Town, South Africa. p. 168.

Strydom, P.E., 2011. Quality related principles of the South African beef classification system in relation to grading and classification systems of the world. S. Afr. J. Anim. Sci. 41, 177-193.

Strydom, P.E., 2013. Beef Quality Audit final report, Red Meat Research and Development SA Project Committee (RMRD S-PC).

Tambor, V., Fucíková, A., Lenco, J., Kacerovský, M., Rehácek, V., Stulík, J. \& Pudil, R., 2010. Application of Proteomics in biomarker discovery: A primer for the clinician. Physiol. Res. 59, 471-497. www.bio-rad.com/en-us/product/pdquest-2-d-analysis-software 